\title{
On the Stability of Finite Difference Schemes Which Approximate Regularly Hyperbolic Systems with Nearly Constant Coefficients
}

\author{
By \\ Yoshinori KaMETAKA*
}

\section{Introduction}

Consider a first order hyperbolic system of partial differential equations

$$
\frac{\partial u}{\partial t}=\sum_{j=1}^{n} A_{j}(x) \frac{\partial u}{\partial x_{j}} \quad(t, x) \in[0, T] \times R^{n}
$$

and initial data at $t=0$

$$
u(0, x)=u_{0}(x) \quad x \in R_{x}^{n} .
$$

We approximate the Cauchy problem (1.1) (1.2) by finite difference scheme

$$
\begin{aligned}
& u(t+k, x)=S_{h} u(t, x) \\
& u(0, x)=u_{0}(x) .
\end{aligned}
$$

Here $k>0$ is a time-step and $h>0$ is a mesh-width, as usual we assume that $k / h=\lambda=$ constant.

We call the approximation (1.3) is stable if $S_{h}^{m}$ is uniformly bounded on $L^{2}\left(R^{n}\right)$ for $0 \leq m h \leq T$.

In constant coefficient case there are many usefull criteria on the stability of finite difference approximation. (Kreiss [3], Parlett [4], Yamaguti [7]).

Received December 1, 1967.

Communicated by S. Matsuura.

* Osaka City University. 
In variable coefficient case Kreiss obtained sufficient condition for stability for symmetric hyperbolic systems. But for non-symmetric hyperbolic systems with variable coefficients there are much less results, except for few special examples (Friedrichs scheme, modified LaxWendroff scheme. (Yamaguti and Nogi [8] ).

We obtained some kind of sufficient conditions for stability of primary type schemes which approximate regularly hyperbolic systems with nearly constant coefficients.

\section{Pseudo Difference Scheme}

To obtain energy inequality for non-symmetric hyperbolic partial differential equations with variable coefficients pseudo differential operators was a very usefull tool. Here following the ideas of Yamaguti and Nogi [8] we introduce pseudo difference scheme to obtain local energy inequality for finite difference schemes which approximate nonsymmetric hyperbolic partial differential equations.

Let $K(x, \xi) \in C^{\infty}\left(R^{n} \times R^{n}-\{0\}\right)$ be homogeneous degree 0 in $\xi$ and $K(x, \xi)=K(\infty, \xi)$ for $|x|>R$. We define pseudo difference scheme $K_{k}$ with symbol $K(x, \xi)$ as follows:

\section{Definition 2.1.}

$$
\begin{aligned}
& K_{k} u(x)=\text { l.i.m. } \int e^{i x \xi} K(x, \sin h \xi) \hat{u}(\xi) d \xi \\
& \hat{u}(\xi)=\mathscr{L} u(\xi)=(2 \pi)^{-n / 2} \int e^{-i x \xi} u(x) d x \\
& \sin h \xi=\left(\sin h \xi_{1}, \cdots, \sin h \xi_{n}\right) .
\end{aligned}
$$

Corresponding to the operator $A$ in the theory of pseudo differential operators we define the operator $\Lambda_{l}$ as follows:

\section{Definition 2.2.}

$$
\begin{aligned}
& \Lambda_{h} u=\mathscr{F}^{-1}|\sin h \xi| \mathscr{F} u \\
& |\sin h \xi|=\left(\left|\sin h \xi_{1}\right|^{2}+\cdots+\left|\sin h \xi_{n}\right|^{2}\right)^{1 / 2} .
\end{aligned}
$$

We call one parameter family of bounded operators $\left\{H_{h} ; h>0\right\}$ null scheme and write $H_{h} \in \mathcal{I}_{0}$ if $\left\|H_{h}\right\|=0(h)$ as $h \rightarrow 0$. 
Following Yamaguti and Nogi we list up some fundamental properties of pseudo difference schemes.

Lemma 2.1. Let $a(x)$ be a smooth function and constant for $|x|>R$. Let $K_{h}, K_{h}^{*}, K_{1, h}$ and $K_{2, h}$ be pseudo difference schemes with symbols $K(x, \xi), K^{*}(x, \xi), K_{1}(x, \xi)$, and $K_{2}(x, \xi)$ respectively and $T^{j}$ be translation operator. Then pseudo difference schemes of following forms are all null schemes.

$$
\begin{aligned}
& a(x) \Lambda_{h}-\Lambda_{h} a(x), K_{h} \Lambda_{h}-\Lambda_{h} K_{h},\left[a(x) K_{k}-K_{h} a(x)\right] \Lambda_{h}, \\
& \left(K_{k}-K_{h}^{\ddagger}\right) \Lambda_{h},\left(K_{1, h} K_{2, h}-K_{1, h} \circ K_{2, h}\right) \Lambda_{k}, K_{h} T^{j}-T^{j} K_{k} .
\end{aligned}
$$

\section{Primary Type Scheme}

We define the primary type scheme correspond to the hyperbolic system (1.1) by

\section{Definition 3.1.}

$$
\begin{aligned}
& S_{l}=\sum_{j=0}^{s}\left\{\lambda A(x) \cdot \frac{T_{h}-T_{h}^{-1}}{2}\right\}^{j} C_{j}\left(T_{h}\right) \\
& A(x) \frac{T_{h}-T_{h}^{-1}}{2}=\sum_{j=1}^{n} A_{j}(x) \frac{T_{j, h}-T_{j, h}^{-1}}{2} .
\end{aligned}
$$

Here $T_{k}=\left(T_{1, k}, \cdots, T_{n, h}\right)$ is translation operator, $C_{j}\left(T_{h}\right)$ are polynomials in $T_{h}$, and $C_{j}\left(e^{i \xi}\right)$ are real valued functions in $\xi \in R^{n}$. Let introduce the pseudo difference scheme $A_{l}$ with symbol

$$
A(x) \cdot \xi^{\prime}=\sum_{j=1}^{n} A_{j}(x) \frac{\xi_{j}}{|\xi|}
$$

and write (3.1)

$$
S_{h}=\sum_{j=0}^{s}\left(i \lambda A_{h} \Lambda_{h}\right)^{j} C_{j, h} .
$$

Assumption 3.1. We assume that (1.1) is regularly hyperbolic system with nearly constant coefficients, that is

$$
A_{j}(x)=A_{\infty, j}+A_{0, j}(x) \quad j=1, \cdots, n .
$$

Here $A_{\infty, j}$ are constants, $A_{0, j}(x)$ are smooth functions with compact supports and $\left|A_{0, j}(x)\right| \mathscr{B}^{\circ}$ are sufficiently small. $A(x) \cdot \xi^{\prime}$ has real distinct eigenvalues $d_{j}(x, \xi) j=1, \cdots, N$ and 


$$
\inf _{\substack{x \in R^{n} \\ \text { and } \\ j \neq k}}\left|d_{j}(x, \xi)-d_{k}(x, \xi)\right|=\delta>0 .
$$

It is well known from the theory of regularly hyperbolic partial differential equations that there exist the diagonalizer $N(x, \xi)$ with following properties

$$
N(x, \xi) A(x) \cdot \xi^{\prime}=D(x, \xi) N(x, \xi) \quad D(x, \xi)=\left(\begin{array}{cc}
d_{1}(x, \xi) & 0 \\
0 & d_{N}(x, \xi)
\end{array}\right)
$$

(3. 8) $\left|N_{\infty}^{-1}(\xi)\right|,\left|N_{\infty}(\xi)\right|,\left|N_{0}(x, \xi)\right| \leq$ constant independent of $x$ and

$\xi$. By $|x| \rightarrow \infty$ we get the relation

$$
N_{\infty}(\xi) A_{\infty} \cdot \xi^{\prime}=D_{\infty}(\xi) N_{\infty}(\xi) \quad D_{\infty}(\xi)=\left(\begin{array}{ccc}
d_{1, \infty}(\xi) & 0 \\
0 & \ddots \cdot & \\
0 & d_{N, \infty}(\xi)
\end{array}\right) .
$$

Here $d_{j, \infty}(\xi)$ are eigenvalues of $A_{\infty} \cdot \xi^{\prime}$ and we put

$$
d_{j}(x, \xi)=d_{j, \infty}(\xi)+d_{j, 0}(x, \xi) \text {. }
$$

Now we call

$$
S(x, \xi)=\sum_{j=0}^{s}(i \lambda A(x) \sin \xi)^{j} C_{j}(\xi)
$$

the symbol (or amplification matrix) of $S_{k}$ of the form (3.1). Here $C_{j}(\xi)$ are abbreviation of $C_{j}\left(e^{i \xi}\right)$. By the spectral mapping theorem eigenvalues of $S(x, \xi)$ are

$$
\sigma_{k}(x, \xi)=\sum_{j=0}^{s}\left\{i \lambda d_{k}(x, \sin \xi)|\sin \xi|\right\}^{j} C_{j}(\xi) \quad k=1, \cdots, N .
$$

\section{Stability}

Theorem 4.1. Suppose that the assumption 3.1 is satisfied and for eigenvalues of the symbol $S(\infty, \xi)$ we assume

$$
\left|\sigma_{k}(\xi)\right|^{2} \leq 1-\delta_{\infty}|\sin \xi|^{2 r} \quad k=1, \cdots, N .
$$

Then the scheme (3.1) is stable if $\lambda$ is sufficiently small and if coefficients $C_{j}(\xi)$ of the symbol $S(x, \xi)$ satisfy following relations

$$
\left|2 \sum_{j=0}^{l-1}(-1)^{j} C_{j}(\xi) C_{2 l-j}(\xi)+(-1)^{l} C_{l}^{2}(\xi)\right| \leq \text { const. }|\sin \xi|^{2(r-l)}
$$




$$
l=1, \cdots, r-1
$$

\section{Accuracy}

Definition 5.1. The difference scheme $S_{k}$ with symbol $S(x, \xi)$ is accurate of order $p$ if

$$
S(x, \xi)=e^{i \lambda A(x) \cdot \xi}+O\left(|\xi|^{p+1}\right) \quad|\xi| \rightarrow 0
$$

Proposition 5.1. Necessary and sufficient condition for that the primary type scheme (3.1) is accurate of order $p$ (uniformly in $\lambda, 0 \leq$ $\left.\lambda \leq \lambda_{0}\right)$ is in the case $p=1$

$$
\left.\begin{array}{l}
C_{0}(\xi)=1+O\left(|\xi|^{2}\right) \\
C_{1}(\xi)=1+O(|\xi|)
\end{array}\right\} \quad|\xi| \rightarrow 0
$$

and in the case $p=2$

$$
\left.\begin{array}{l}
C_{0}(\xi)=1+O\left(|\xi|^{3}\right) \\
C_{1}(\xi)=1+O\left(|\xi|^{2}\right) \\
C_{2}(\xi)=1+O(|\xi|)
\end{array}\right\} \quad|\xi| \rightarrow 0 .
$$

Remark 5.1. In general it is impossible to obtain the primary type scheme $S_{i}$ of the form (3.1) with accuracy $p \geq 3$.

\section{Proof of Theorem 4.1}

Let $N_{h}$ be pseudo difference scheme with symbol $N(x, \xi)$ the diagonalizer of $A(x) \cdot \xi^{\prime}$. We introduce new norm on $L^{2}\left(R^{n}\right)$ by use of $N_{h}$ which is equivalent to usual $L^{2}$-norm.

Lemma 6.1. Under the assumption 3.1 we have

$$
\delta_{1}\|u\| \leq\left\|N_{h} u\right\| \leq \delta_{2}\|u\| \quad \forall u \in L^{2}\left(R^{n}\right) .
$$

Here $\delta_{1}$ and $\delta_{2}$ are constants independent of $h>0$.

Let $N_{\infty, h}$ and $N_{0, h}$ be pseudo difference scheme with symbols $N_{\infty}(\xi)$ and $N_{0}(x, \xi)$ respectively. We have

$$
N_{h}=N_{\infty, h}+N_{0, h} .
$$


Remember that $N_{0}(x, \xi)$ is homogeneous degree 0 in $\xi$ and can be expanded by means of spherical harmonics

$$
N_{0}(x, \xi)=\sum_{l, m} n_{0, l, m}(x) Y_{l, m}(\xi) \text {. }
$$

Here $\left\{Y_{l, m}(\xi)\right\}_{m=1, \cdots, n(l)}$ is a base of spherical harmonics of degree $l$. Following properties are well known (Calderón and Zygmund [1]):

$$
\begin{aligned}
& \left|Y_{l, m}(\xi)\right| \leq \text { const. } l^{(n-2) / 2} \\
& n(l) \leq \text { const. } l^{n-2}
\end{aligned}
$$

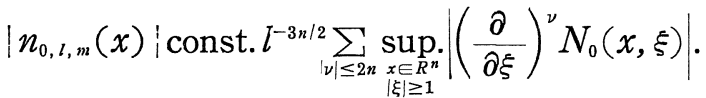

By (6.4), (6.5) and (6.6) the expansion (6.3) converges absolutely and uniformly in $(x, \xi) \in R^{n} \times\left\{\xi \in R^{n} ;|\xi|=1\right\}$. Therefore pseudo difference scheme $N_{0, h}$ can be expressed as

$$
N_{0, h} u=\sum_{l, m} n_{0, l, m}(x) \mathscr{I}^{-1}\left[Y_{l, m}(\sin h \xi) \hat{u}(\xi)\right] .
$$

Proof of Lemma 6.1.

is obvious.

$$
\left\|N_{h} u\right\| \leq \delta_{2}\|u\|
$$

$$
\begin{aligned}
& \left\|N_{h} u\right\| \geq\left\|N_{\infty, h} u\right\|-\left\|N_{0, h} u\right\| \\
& =\left\|N_{\infty}(\sin h \xi) \hat{u}(\xi)\right\|-\left\|\sum_{l, m} n_{0, l, m}(x) \mathscr{F}^{-1}\left[Y_{l, m}(\sin h \xi) \hat{u}(\xi)\right]\right\| \\
& \geq 2 \delta_{1}\|u\|-\sum_{l, m}\left|n_{0, l, m}(x)\right| \mathscr{B}^{0}\left|Y_{l, m}(\xi)\right| \mathscr{B}^{0}\|u\| \\
& \geq\left\{2 \delta_{1}-\text { const. } \sum_{\substack{|\nu| \leq 2 n \\
\sup _{x \in R^{n}}}} \mid\left(\frac{\partial}{\partial \xi \mid \geq 1}\right)^{\nu} N_{0}(x, \xi)\right\}\|u\| \text {. }
\end{aligned}
$$

In assumption 3.1 if we take $\left|A_{0}(x)\right| \mathscr{B}^{0}$ sufficiently small, we can assume

$$
\text { const. } \sum_{|\nu| \leq 2 n} \sup _{\substack{x \in R^{n} \\|\xi| \geq 1}}\left|\left(\frac{\partial}{\partial \xi}\right)^{\nu} N_{0}(x, \xi)\right|<\delta_{1} .
$$

Therefore we obtain

$$
\left\|N_{h} u\right\| \geq \delta_{1}\|u\| \quad \forall u \in L^{2}\left(R^{n}\right) .
$$

This completes the proof of lemma 6.1.

Proof of Theorem 4.1. It is sufficient for the stability of the 
scheme (3.1) to show that

$$
\left\|N_{h} S_{h} u\right\|^{2} \leq\{1+O(h)\}\left\|N_{h} u\right\|^{2} \quad \forall u \in L^{2}\left(R^{n}\right) .
$$

By use of lemma 2.1 we can calculate $N_{h} S_{h}$ modulo null scheme $\Omega_{0}$ as

$$
\begin{aligned}
& N_{h} S_{h}=\sum_{j=0}^{s} N_{i h}\left(i \lambda A_{h} \Lambda_{h}\right)^{j} C_{j, h} \equiv \sum_{j=0}^{s}\left(i \lambda D_{i h} \Lambda_{h}\right)^{j} N_{h} C_{j, h} \\
& \equiv \sum_{j=0}^{s}\left(i \lambda D_{h} \Lambda_{h}\right)^{j} C_{j, h} N_{h \cdot} .
\end{aligned}
$$

The inequality (6.8) is reduced to more simple form

$$
\left[\left(1-\left\{\sum_{j=0}^{s}\left(i \lambda d_{h} \Lambda_{k}\right)^{j} C_{j, h}\right\}^{*}\left\{\sum_{k=0}^{s}\left(i \lambda d_{h} \Lambda_{h}\right)^{k} C_{k, h}\right\}\right] v, v\right) \geq-O(h)\|v\|^{2}
$$

for any scalar valued function $v \in L^{2}\left(R^{n}\right)$. Here $d_{h}$ is pseudo difference scheme whose symbol is any one of eigenvalues $d_{j}(x, \xi)$ of $A(x) \cdot \xi^{\prime}$. Let $j+k=2 l+1$, where $j, k$ and $l$ are non-negative integers, therefore $j$ is even and $k$ is odd or conversely $j$ is odd and $k$ is even.

$$
\begin{aligned}
& C_{j, h}\left(i \lambda d_{h} \Lambda_{h}\right)^{* j}\left(i \lambda d_{h} \Lambda_{h}\right)^{k} C_{k, h}+C_{k, h}\left(i \lambda d_{k} \Lambda_{h}\right)^{* k}\left(i \lambda d_{h} \Lambda_{h}\right)^{j} C_{j, h} \\
& \equiv C_{j, h}\left(-i \lambda d_{i k} \Lambda_{h}\right)^{j}\left(i \lambda d_{h} \Lambda_{h}\right)^{k} C_{k, h}+C_{k, h}\left(-i \lambda d_{k} \Lambda_{k}\right)^{k}\left(i \lambda d_{k} \Lambda_{k}\right)^{j} C_{j, h} \\
& \equiv\left\{(-1)^{j}+(-1)^{k}\right\}\left(i \lambda d_{k} \Lambda_{k}\right)^{j+k} C_{j, h} C_{k, h}=0 .
\end{aligned}
$$

Next in the case $j+k=2 l, 0 \leq j \leq l-1$

$$
\begin{aligned}
& C_{j, h}\left(i \lambda d_{i t} \Lambda_{h}\right)^{* j}\left(i \lambda d_{h} \Lambda_{i h}\right)^{k} C_{k, h}+C_{k, h}\left(i \lambda d_{h} \Delta_{h}\right)^{* k}\left(i \lambda d_{h} \Lambda_{h}\right)^{j} C_{j, h} \\
& \equiv(-1)^{l}\left(\lambda d_{h} \Lambda_{h}\right)^{2 l} 2(-1)^{j} C_{j, h} C_{2 l-j, h}
\end{aligned}
$$

and in the case $j=k=l$

$$
C_{l, h}\left(i \lambda d_{k} \Lambda_{l l}\right)^{* l}\left(i \lambda d_{h} \Lambda_{k l}\right)^{l} C_{l, h} \equiv(-1)^{l}\left(\lambda d_{h} \Lambda_{h}\right)^{2 l}(-1)^{l} C_{l, h}^{2}
$$

Thus we have

$$
\begin{aligned}
& \left\{\sum_{j=0}^{s}\left(i \lambda d_{h} \Lambda_{h}\right)^{j} C_{j, h}\right\}^{*}\left\{\sum_{k=0}^{s}\left(i \lambda d_{l} \Lambda_{h}\right)^{k} C_{k, h}\right\} \\
& \quad \equiv \sum_{l}(-1)^{l}\left(\lambda d_{l} \Lambda_{k}\right)^{2 l}\left\{2 \sum_{j=0}^{l-1}(-1)^{j} C_{j, h} C_{2 l-j, k}+(-1)^{l} C_{l, h}^{2}\right\} .
\end{aligned}
$$

Let $d_{\infty, h}$ and $d_{0, h}$ be pseudo difference schemes with symbols $d_{\infty}(\xi)$ and $d_{0}(x, \xi)$ respectively. We have 


$$
\begin{aligned}
d_{h} & =d_{\infty, h}+d_{0, h} \\
1- & \left\{\sum_{j=0}^{s}\left(i \lambda d_{h} \Lambda_{h}\right)^{j} C_{j, h}\right\}^{*}\left\{\sum_{k=0}^{s}\left(i \lambda d_{h} \Lambda_{h}\right)^{k} C_{k, h}\right\} \\
& \equiv 1-\left\{\sum_{j=0}^{s}\left(i \lambda d_{\infty, h} \Lambda_{h}\right)^{j} C_{j, h}\right\}^{*}\left\{\sum_{k=0}^{s}\left(i \lambda d_{\infty h} \Lambda_{h}\right)^{k} C_{k, h}\right\} \\
& -\sum_{l \geq 1}(-1)^{l}\left\{\left(\lambda d_{h} \Lambda_{h}\right)^{2 l}-\left(\lambda d_{\infty, h} \Lambda_{h}\right)^{2 l}\right\}\left\{2 \sum_{j=0}^{l-1}(-1)^{j} C_{j, h} C_{2 l-j, h}+(-1)^{l} C_{l, h}^{2}\right\} .
\end{aligned}
$$

From the assumption (4.1) of theorem 4.1 we obtain the estimate

$$
\begin{aligned}
& \left(\left[1-\left\{\sum_{j=0}^{s}\left(i \lambda d_{\infty, h} \Lambda_{h}\right)^{j} C_{j, h}\right\}^{*}\left\{\sum_{k=0}^{s}\left(i \lambda d_{\infty, h} \Lambda_{h}\right)^{k} C_{k, h}\right\} v, v\right)\right. \\
& \quad=\left(\left\{1-\left|\sigma_{\infty}(h \xi)\right|^{2}\right\} \hat{v}, \hat{v}\right) \\
& \quad \geq \delta\left(|\sin h \xi|^{2 r} v, \hat{v}\right)=\delta\left\|\Lambda_{h}^{r} v\right\|^{2} .
\end{aligned}
$$

On the other hand

$$
\begin{aligned}
& \left(\lambda d_{h} \Lambda_{h}\right)^{2 l}-\left(\lambda d_{\infty, h} \Lambda_{h}\right)^{2 l} \equiv \lambda^{2 l}\left\{\left(d_{\infty, h}+d_{0, h}\right)^{2 l}-d_{\infty, h}^{2 l}\right\} \Lambda_{h}^{2 l} \\
& \equiv \lambda^{2 l} d_{0, h} \circ\left\{\left(d_{\infty, h}+d_{0, h}\right)^{2 l-1}+\cdots+d_{\infty, h}^{2 l-1}\right\} \Lambda_{h}^{2 l} .
\end{aligned}
$$

If $l \leq r-1$ by the assumption of theorem 4.1 , we have

$$
\left|2 \sum_{j=0}^{l-1}(-1)^{j} C_{j}(h \xi) C_{2 l-j}(h \xi)+(-1)^{l} C_{l}^{2}(h \xi)\right| \leq \text { const. }|\sin h \xi|^{2(r-l)} .
$$

Finally by taking $\lambda$ and $\left|A_{0}(x)\right| \mathscr{B}^{\circ}$ sufficiently small we have

$$
\begin{aligned}
& \mid\left(\sum _ { l \geq 1 } ( - 1 ) ^ { l } \{ ( \lambda d _ { h } \Lambda _ { h } ) ^ { 2 l } - ( \lambda d _ { \infty , h } \Lambda _ { h } ) ^ { 2 l } \} \left\{2 \sum_{j=0}^{l-1}(-1)^{j} C_{i, h} C_{2 l-j, h}\right.\right. \\
& \left.\left.\quad+(-1)^{l} C_{l, h}^{2}\right\} v, v\right) \mid \leq \frac{\delta}{2}\left\|\Lambda_{h}^{r} v\right\|^{2} .
\end{aligned}
$$

We obtain therefore the desired estimate (6.9). This completes the proof of theorem 4.1 .

Remark 6.1. We can replace the inequality (4.1) by

$$
\begin{aligned}
& 1-\sum_{l}(-1)^{l}\left(\lambda d_{\infty}(\sin \xi)|\sin \xi|\right)^{2 l}\left\{2 \sum_{j=0}^{l-1}(-1)^{j} C_{j}(\xi) C_{2 l-j}(\xi)\right. \\
& \left.+(-1)^{l} C_{l}^{2}(\xi)\right\} \geq \text { const. }|\sin \xi|^{2 r} .
\end{aligned}
$$

\section{Schemes with High Order of Accuracy}

If we wish to consider schemes with higher order of accuracy we 
must replace $\sin \xi$ by

$$
\begin{aligned}
& s_{q}(\xi)=\sum_{l=1}^{q} \gamma_{2 l-1}(\sin \xi)^{2 l-1}=\xi+O\left(|\xi|^{2 q+1}\right) \quad|\xi| \rightarrow 0 \\
& \gamma_{2 l-1}=\frac{(2 l-2) !}{(2 l-1) 2^{2 l-2}((l-1) !)^{2}} .
\end{aligned}
$$

Now the finite difference scheme with symbol of the following form we shall again call primary type.

$$
\begin{aligned}
& S(x, \xi)=\sum_{j=0}^{s}\left(i \lambda A(x) \cdot s_{q}(\xi)\right)^{j} C_{j}(\xi) \\
& s_{q}(\xi)=\left(s_{q}\left(\xi_{1}\right), \cdots, s_{q}\left(\xi_{n}\right)\right) .
\end{aligned}
$$

We must change the definition of pseudo difference scheme.

\section{Definition 7.1.}

$$
\begin{aligned}
& \Lambda_{h} u=\mathscr{F}^{-1}\left[\left|s_{q}(h \xi)\right| \hat{u}(\xi)\right] \\
& \left|s_{q}(\xi)\right|=\left(\left|s_{q}\left(\xi_{1}\right)\right|^{2}+\cdots+\left|s_{q}\left(\xi_{n}\right)\right|^{2}\right)^{1 / 2} .
\end{aligned}
$$

\section{Definition 7.2.}

$$
K_{k} u=1 . \mathrm{i} . \mathrm{m} . \int e^{i x \xi} K\left(x, s_{q}(h \xi)\right) \hat{u}(\xi) d \xi .
$$

Fundamental properties of pseudo difference schemes of section 2 remain unchanged.

Proposition 7.1. Let $p \leq 2 q$, necessary and sufficient condition for that the finite difference scheme $S_{k}$ with symbol (7.2) is accurate of order $p$ uniformly in $\lambda, 0 \leq \lambda \leq \lambda_{0}$, is

$$
C_{j}(\xi)=\frac{1}{j !}+O\left(|\xi|^{p+1-j}\right) \quad j=0,1, \cdots, p .
$$

Theorem 7.1. The finite difference scheme $S_{h}$ with symbol (7.2) is stable under the same assumption of theorem 4.1.

Proof is quite similar to that of theorem 4.1. It is sufficient only to see

$$
\text { const. }|\sin \xi| \leq\left|s_{q}(\xi)\right| \leq \text { const. }|\sin \xi| \text {. }
$$




\section{Remarks on Symmetric Hyperbolic Case}

Let (1.1) be symmetric hyperbolic system with variable coefficients, that is, we assume that $A_{j}(x)$ are all hermitian $N \times N$ matrices. As before we approximate the Cauchy problem (1.1) (1.2) by finite difference scheme (1.3) (1.4). Here $S_{l}$ is again the primary type scheme of the form (3.1).

Theorem 8.1. In this case the von Neumann condition is necessary and sufficient for stability.

Proof. Necessity of the von Neumann condition for finite difference scheme with variable coefficients is due to $G$. Strang [5]. It is sufficient for stability to establish the inequality

$$
\left(\left(I-S_{h}^{*} S_{h}\right) u, u\right) \geq-O(h)\|u\|^{2} \quad \forall u \in L^{2}\left(R^{n}\right) .
$$

Neglecting null scheme we can regard $I-S^{*}(x, \xi) S(x, \xi)$ is the symbol (in the sense of Lax-Nirenberg [6]) of $I-S_{h}^{*} S_{k}$. In this case $S^{*}(x, \xi)$ $S(x, \xi)$ and $S(x, \xi)$ are diagonalized in the same time by unitary matrix and consequently $\sigma\left(S^{*} S\right)=|\sigma(S)|^{2}$. Here $\sigma(S)$ means eigenvalue of matrix $S$. Therefore the von Neumann condition

$$
|\sigma(S(x, \xi))| \leq 1 \quad \forall(x, \xi) \in R^{n} \times R^{m}
$$

assures that the hermitian matrix $I-S^{*}(x, \xi) S(x, \xi)$ is non-negative. In this case $S(x, \xi)$ is trigonometric polynomial in $\xi$. By the theorem of Lax-Nirenberg [6] we can establish the desired inequality (8.1). The proof of the theorem is complete.

\section{Remarks on Regularly Hyperbolic System of One Space Variable}

Let (1.1) be regularly hyperbolic system of one space variable $(n=1)$. There exists the diagonalizer $N(x)$ of $A(\mathrm{x})$ such that

$$
\begin{gathered}
N(x) A(x)=D(x) N(x) \quad D(x)=\left(\begin{array}{cc}
d_{1}(x) & 0 \\
& \ddots \\
0 & d_{N}(x)
\end{array}\right) \\
d_{1}(x), \cdots, d_{N}(x) \text { are eigen values of } A(x)
\end{gathered}
$$




$$
\left|N^{-1}(x)\right|,|N(x)| \leq \text { const. }
$$

By multiplying $\xi \in R^{1}$ on both sides of (9.1) we get

$$
N(x) A(x) \cdot \xi=D(x) \cdot \xi N(x) \text {. }
$$

In this case $\|N(x) u\|$ defines an equivalent norm on $L^{2}\left(R^{n}\right)$ without the assumption of nearly constant coefficients.

Theorem 9.1. In this case the von Neumann condition is necessary and sufficient for the stability of the scheme of the primary type (3. 1).

Proof. Attention that the symbol

$$
\sum_{j=0}^{s}\left\{i \lambda d_{k}(x) \sin \xi\right\}^{j} C_{j}(\xi)
$$

is trigonometric polynomial in $\xi$ and the theorem of Lax-Nirenberg is applicable.

\section{Examples}

Example 10.1. The main theorem 4.1 is applicable for Friedrichs scheme

$$
S(x, \xi)=\frac{1}{n} \sum_{j=1}^{n} \cos \xi_{j}+i \lambda A(x) \cdot \sin \xi
$$

and modified Lax-Wendroff scheme

$$
S(x, \xi)=I+i \lambda A(x) \cdot \sin \xi \frac{1}{n} \sum_{j=1}^{n} \cos \xi_{j}+\frac{1}{2}(i \lambda A(x) \cdot \sin \xi)^{2} .
$$

But in these cases there are more sharper results by Yamaguti and Nogi [8].

\section{Example 10.2.}

$$
\begin{aligned}
& S(x, \xi)=I+i \lambda A(x) \cdot \sin \xi+\frac{1}{2}(i \lambda A(x) \cdot \sin \xi)^{2} \\
& \quad+C_{3}(i \lambda A(x) \cdot \sin \xi)^{3} .
\end{aligned}
$$

Let assume that $C_{3}=$ const. $>\frac{1}{8}, \inf _{|\xi|=1}\left|d_{j, \infty}(\xi)\right|>0$. Then this scheme is accurate of order 2 and

$$
\left|\sigma_{k, \infty}(\xi)\right|^{2} \leq 1-\delta_{\infty}|\sin \xi|^{4}
$$




$$
2 C_{0} C_{2}-C_{1}^{2}=0 \text {. }
$$

Therefore this scheme is stable under the assumption 3.1 and if $\lambda$ is small enough.

\section{References}

[1] Calderón, A. P. and A. Zygmund, Singular integral operators and differential equations, Amer. J. Math. 79 (1957), 901-921.

[2] Mizohata, S., Systèmes hyperboliques, J. Math. Soc. Japan, 11 (1959), 205-233.

[3] Kreiss, H. O., On difference approximations of the dissipative type for hyperbolic differential equations, Comm. Pure Appl. Math. 17 (1964), 335-353.

[4] Parlett, B. N., Accuracy and dissipation in difference schemes, Comm. Pure Appl. Math. 19 (1966), 111-123.

[5] Strang, G., Necessary and insufficient conditions for well-posed Cauchy problems, J. Differential Equations, 2 (1966), 107-114.

[6] Lax, P. D. and L. Nirenberg, On stability for difference schemes; a sharp form of Gårding's inequality, Comm. Pure Appl. Math. 19 (1966), 473-492.

[7] Yamaguti, M., Some remarks on the Lax-Wendroff finite difference scheme for non-symmetric hyperbolic systems, Math. Comp. 21 (1967).

[8] Yamaguti, M., and T. Nogi, An algebra of pseudo difference schemes and its application, Publ. RIMS Kyoto Univ. Ser. A, 3 (1967), 151-166. 